\title{
Gasless Endoscopic Thyroidectomy Using Trans-axillary Approach; Surgical Outcome of 581 Patients
}

\author{
Sang-Wook KANG, Jong Ju JEONG, Ji-Sup YUN*, Tae Yon SUNG, Seung Chul LEE, Yong Sang LEE, \\ Kee-Hyun NAM, Hang Seok CHANG, Woong Youn Chung, Cheong Soo PARK \\ Department of Surgery, Yonsei University College of Medicine, C.P.O. Box 8044, 250 Seongsanno, Seodaemun-gu, Seoul 120-752, \\ South Korea \\ *Department of Surgery, Sungkyunkwan University, School of Medicine, Kangbuk Samsung Hospital, 108 Pyung-dong, Jongno-Ku, \\ Seoul, South Korea
}

\begin{abstract}
Purpose: During the past decade, various techniques of endoscopic thyroid surgery have been introduced. We have developed a novel method of gasless endoscopic thyroidectomy via an axillary approach. The present report describes the technique of this method, and analyzes its surgical outcome.

Patients and Methods: Between Nov. 2001 and Dec. 2007 (actual operation period was 50 months), 581 patients with thyroid tumors underwent gasless endoscopic thyroidectomy via an axillary approach. The clinical and pathologic characteristics of patients, operation type, operation time, post operative hospital stay and post operative complications were analyzed retrospectively.

Results: Among the 581 patients, 171 patients had benign tumor and 410 patients had malignant tumor. There was no conversion to open surgery. The operating time and the length of post-operative hospital stay were $129.4 \pm 51.3$ minutes, $3.3 \pm 1.7$ days in benign tumor, and $135.5 \pm 47$ minutes, $3.4 \pm 0.9$ days in malignant tumors, respectively. The tumor size was $2.7 \pm 1.2 \mathrm{~cm}$ in benign tumor and $0.78 \pm 0.5 \mathrm{~cm}$ in malignancy. Central compartment lymph node metastasis was found in $112(27.3 \%)$ patients and lateral neck lymph node metastasis in $13(3.1 \%)$ patients. As post-operative complications, transient hypocalcemia occurred in 19 patients and transient hoarseness was in 13 patients and permanent vocal cord palsy occurred in 2 patients. In the TNM stage, $366(89.2 \%)$ patients were stage I, $43(10.5 \%)$ patients were stage III and 1 $(0.2 \%)$ patient was stage IVA.

Conclusion: According to our experience, gasless endoscopic thyroidectomy using a trans-axillary approach is a feasible and safe method. Endoscopic thyroid surgery has become a new treatment modality for the patients with benign tumors and can be an effective alternative treatment for the selected patients with thyroid cancer.
\end{abstract}

Key words: Endoscopic thyroid surgery, Gasless, Trans-axillary approach

(Endocrine Journal 56: 361-369, 2009)

FROM the beginning of the twenty first century, people have been greatly interested in the sense of "well-being". This trend has been applied not only in the medical parts but also in surgical areas, such as post operative pain degree, cosmetic problems of operation scar, and time it takes to be able to work again. Thyroid disease usually occurs in women and

Received: October 29, 2008

Accepted: December 25, 2008

Correspondence to: Woong Youn CHUNG, Department of Surgery, Yonsei University College of Medicine, 250 Seongsanno, Seodaemun-gu, Seoul, Korea the incidence of thyroid disease in young women is increasing. As a result, the cosmetic viewpoint is being focused. Conventional open thyroidectomy usually have a long incision line $(6 \sim 8 \mathrm{~cm})$ on the anterior neck where it is always opened, and sometimes leaves a prominent scar on the anterior neck. This point was getting troublesome to both patients and doctors. Minimally invasive surgeries including endoscopic operations were developed to overcome this obstacle.

Just after the first report of endoscopic parathyroidectomy by Gagner [1] in 1996 and video-assisted thyroid lobectomy by Hüscher [2] in 1997, various methods of endoscopic thyroid surgery have been in- 
troduced with the development of laparoscopic operation and endoscopic instruments during the past decade [3-6]. We have already reported early experience of gasless endoscopic thyroidectomy using trans-axillary approach [7]. In this paper, we want to know the safety and effectiveness of endoscopic thyroidectomy for patients with thyroid tumors including malignant ones by analyzing the surgical outcome of 581 patients.

\section{Patients and Methods}

\section{Patients}

From Nov. 2001 to Dec. 2007 (during 50 months, excluded 24 months from Sep. 2003 to Aug. 2005 due to surgeon's overseas training), a total of 581 patients who underwent gasless endoscopic thyroidectomy using trans-axillary approach enrolled in this study. Preoperative diagnoses of thyroid nodule were made by ultrasonography guided fine needle aspiration biopsy (FNAB). In the cases of malignancy, the patients were evaluated for tumor size, the fact of capsular invasion, multiplicity, bilaterality, and neck node metastasis.

The inclusion criteria for endoscopic thyroid surgery were as follows: 1) patients who have thyroid tumor not larger than $5 \mathrm{~cm}$ and diagnosed as follicular neoplasm by the FNAB, 2) papillary thyroid microcarcinoma (PTMC) patients with low risk. All the patients who were identified as with wide or definite extra-capsular soft tissue invasion, multiple lateral neck node metastasis or peri-nodal infiltration of metastatic lymph node, and distant metastasis through the pre-operative imaging study were excluded. And the cases where the lesion was located at the posterior capsule area (dorsal surface) of the thyroid, especially adjacent to the tracheo-esophageal (T-E) groove were also excluded to prevent possible injury of the trachea, esophagus and recurrent laryngeal nerve (RLN). The advanced cases such as conglomerated metastatic lymph nodes on the central compartment with adhesion or RLN invasion were excluded. The patients who had a multiple and bilateral lesion, along with a thyroid capsular invasion which was identified during operation, underwent total thyroidectomy. Prophylactic ipsilateral central compartment node dissection (CCND) was done for most of the malignant tumor cases, and modified radical neck dissection was done limitedly for the case of only 1 2 lateral neck node metastasis without the evidence of peri-nodal infiltration by the pre-operative imaging study

We analyzed the patient's clinical characteristics, operation type, operation time, post operative hospital stay, complications, pathologic characteristics (tumor size, invasion of thyroid capsule, multiplicity and bilaterality of tumor, and lateral neck node metastasis) and TNM stage according to the $6^{\text {th }}$ edition of American Joint Committee on Cancer, and International Union Against Cancer (AJCC/UICC) based on the prospectively enrolled data base [8]. All the malignant tumor patients were given levothyroxine for the thyroid stimulation hormone (TSH) suppression after the operation and regularly followed up at a 3 or 6 months interval. The patients, who satisfied the indication of radio-active iodine (RAI) therapy, underwent this procedure in the $4 \sim 6^{\text {th }}$ weeks after the total thyroidectomy. The ${ }^{131} \mathrm{I}$ whole body scan was taken on the second day after the RAI therapy. The mean follow up period was 22.5 (range 9 82) months.

\section{Operation method}

Patients are placed in a supine position while under general anesthesia. The neck is slightly extended, and the lesion-side arm is raised and fixed for the shortest distance from axilla to anterior neck. A $4 \sim 6 \mathrm{~cm}$ sized vertical skin incision is made in the axilla for the insertion of a 45-degree endoscope and endoscopic instruments. The route to the anterior neck area is dissected through the anterior surface of the pectoralis major muscle using an electrical cautery under direct vision, until the anterior border of the sternocleidomastoid muscle (SCM) is exposed. In the beginning, exposition of the thyroid was made through the space between the medial border of SCM and lateral border of the strap muscles. However, since 2006, dissection has been approached through the space of SCM branches (between sternal head and clavicular head) and beneath the strap muscle until the contra-lateral lobe of the thyroid is exposed. An external retractor is inserted through the skin incision in the axilla and the flap is raised using a lifting device to keep a working space. A second skin incision $(0.5 \mathrm{~cm}$ in length) is made on the medial side of the anterior chest wall for the insertion of endoscopic instruments on an imaginary horizontal line starting from the lower end of the axillary incision, extending from 5 to $6 \mathrm{~cm}$. The operation is preceded as the same method of convention- 
(a)

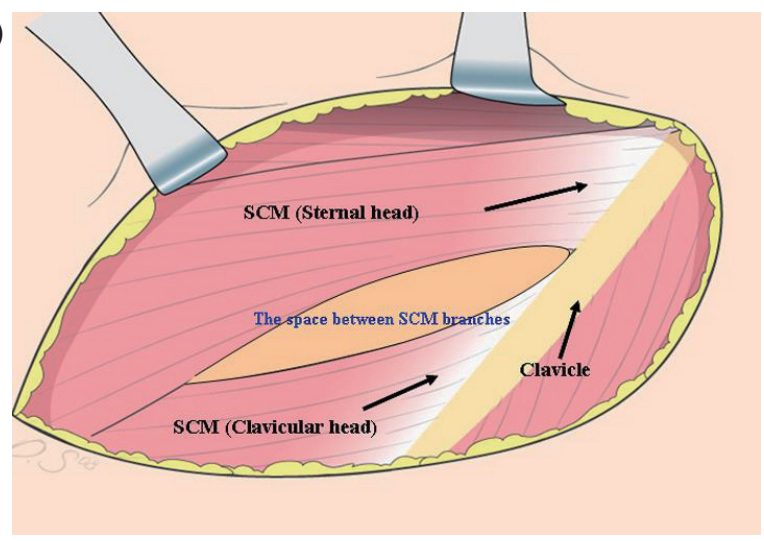

(b)

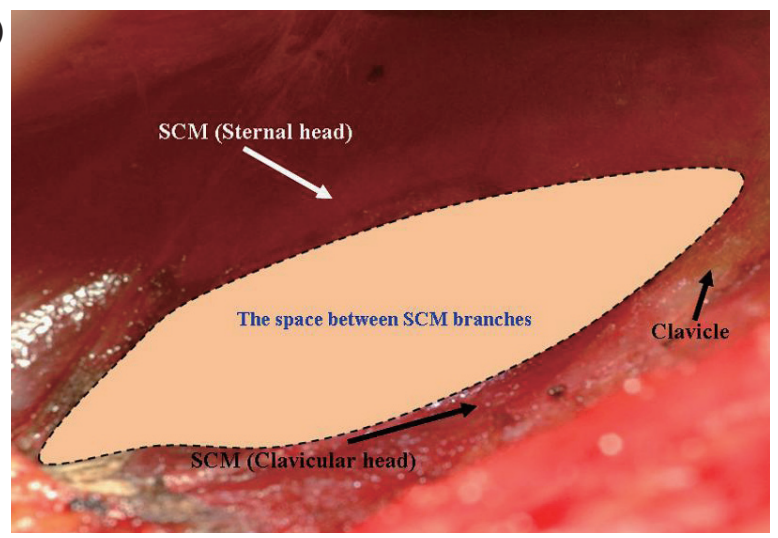

Fig. 1a, b. Approaching route of avascular space between the SCM branches in the right side, (a) illustration image, (b) operative view.

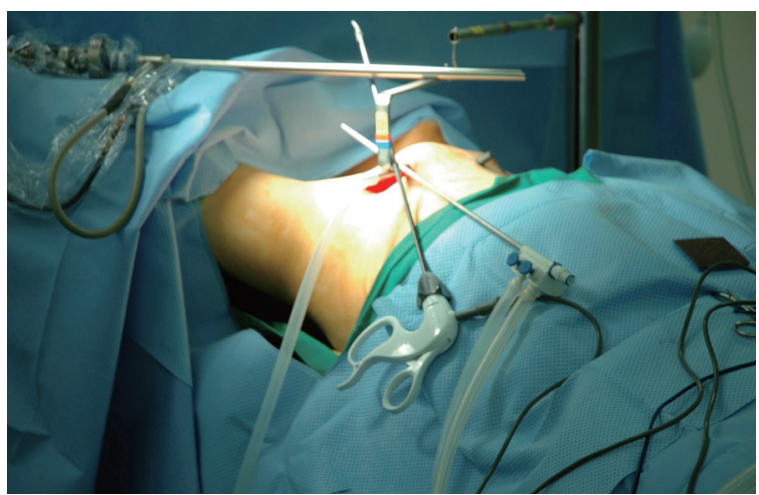

Fig.2. The patient preparation after flap dissection and insertion of external retractor

al open thyroidectomy. Under the endoscopic guidance, the upper pole of the thyroid is drawn downward and the superior thyroid vessels are identified and individually divided close to the thyroid gland to avoid injuring the external branch of the superior laryngeal nerve using the Harmonic Scalpel (Johnson \& Johnson Medical, Cincinnati, OH, USA). The lower pole is dissected from the adipose tissue and cervical thymic tissue, and the inferior thyroid vein is divided close to the thyroid gland for mobilization. The thyroid gland is then retracted medially, and the perithyroidal fascia is divided and sharply dissected using an endoscopic dissector. Careful dissection is performed to identify the inferior thyroid artery and the RLN in the usual anatomic relationship. The inferior thyroid artery is divided close to the thyroid gland using the Harmonic Scalpel, and the whole cervical course of the RLN is traced. The superior parathyroid gland is identified during the dissection and is left intact. The thyroid gland is then dissected from the trachea. The contralateral thyroidectomy is performed using the same method with the antero-medial traction of the thyroid. When CCND (the prelaryngeal, pretracheal, and paratracheal area of the tumor side) is required, while monitoring the RLN track, soft tissue and lymph nodes are detached from the thyro-thymic ligament area with the superior-medial retraction of the thyroid. Dissection, close to the RLN, is performed using a fine endoscopic dissector to avoid damage from the collateral energy of the Harmonic Scalpel.

In the case of modified radical neck dissection (MRND, usually perform postero-lateral neck dissection), the same approach method is used and subplatysmal dissection is made to the midline of the anterior neck, the submandibular gland superiorly, and the trapezius muscle posterioly. At first, dissection of the soft tissue and lymph nodes around the anterior and posterior aspect of the SCM is performed and the carotid artery and internal jugular vein are exposed. Then, the retractor is placed under the SCM, the dissection starts from the anterior aspect of the carotid artery and the jugular vein, and proceeds until the posterior aspect of the internal jugular vein is fully exposed. After that, the dissection of level $V_{B}$ in the posterior neck area is preceded preserving the spinal accessory nerve, the phrenic nerve, the thoracic duct, and the brachial nerve plexus. The dissection is preceded upwardly to level II. The resected specimen is extracted through the $4 \sim 6 \mathrm{~cm}$ skin incision. A 3-mm closed suction drain is inserted through a separate skin incision under the axillary skin incision. The wound is closed cosmetically. 
(a)

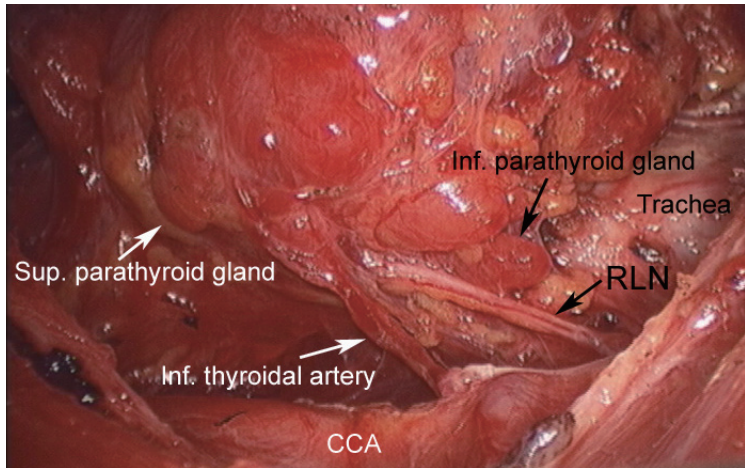

(b)

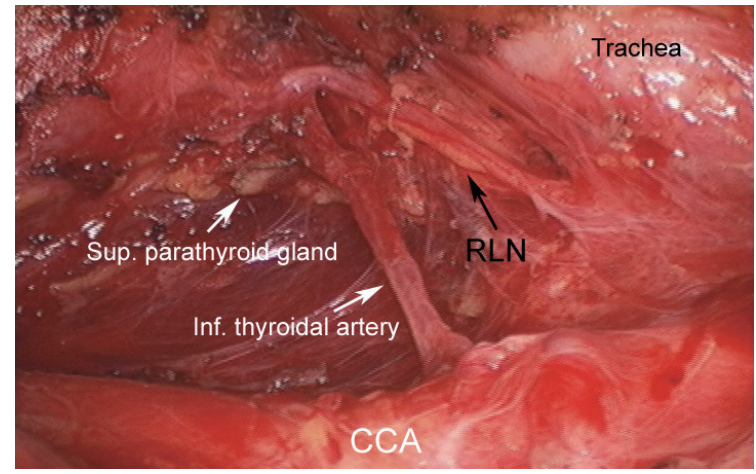

Fig. 3a, b. These pictures show the operative view and anatomic landmarks (a) during surgery, (b) after specimen delivery out.

Table 1. Number and rate of benign, malignant tumor patients according to the period

\begin{tabular}{cccc}
\hline Period & Benign $(N)$ & Malignancy $(N)$ & Rate (benign:malignancy) \\
Nov. 2001 $\sim$ Aug. 2003 & 63 & 48 & $1.3: 1$ \\
Sep. 2005 $\sim$ Aug. 2006 & 49 & 74 & $1: 1.5$ \\
Sep. 2006 Dec. 2008 & 59 & 288 & $1: 4.9$ \\
Total & 171 & 410 & $1: 2.4$ \\
\hline
\end{tabular}

\section{Results}

Among the 581 patients, 171 patients had benign tumors and 410 patients had malignant tumors (benign to malignant ratio was 1:2.4). With experience, the benign to malignant ratio was increased (Table 1). All the cases were successfully performed with endoscopic surgery. The average age of the patients in this study was $36.9 \pm 9.9$ (range 6 65) years and male to female ratio was 1:57.1 (10:571 patients) (Table2).

In 171 benign tumor patients, 139 patients underwent unilateral lobectomy and isthmusectomy, $31 \mathrm{pa}-$ tients ipsilateral total and contralateral subtotal thyroidectomy, and 1 patient total thyroidectomy. In 410 malignant tumor patients, except initial 30 patients, all the patients underwent prophylactic ipsilateral CCND. Unilateral lobectomy and isthmusectomy was performed on 133 patients, ipsilateral total and contralateral subtotal thyroidectomy was performed on 200 patients, and total thyroidectomy was done on 77 patients. In the pre-operative imaging study, there were 26 patients who were suspicious of lateral neck node metastasis. Intraoperative frozen biopsy was performed and 13 patients were diagnosed as lateral neck node metastasis. Among them, 11 patients underwent MRND and 2 patients underwent selective neck dissection (SND) (Table 3).
Table 2. Patient age distribution (mean age 36.9 \pm 9.9 , range 6 65)

\begin{tabular}{cc}
\hline Age & $\mathrm{N}(\%)$ \\
\hline$<10$ & $1(0.17)$ \\
$11 \sim 20$ & $17(2.9)$ \\
$21 \sim 30$ & $157(27.0)$ \\
$31 \sim 40$ & $202(34.7)$ \\
$41 \sim 50$ & $148(25.4)$ \\
$51 \sim 60$ & $53(9.1)$ \\
$>61$ & $3(0.51)$ \\
\hline
\end{tabular}

The operation time of the 171 benign tumor patients was $129.4 \pm 51.3$ (range 50 365) minutes. The operation time of malignant tumor patients was $135.5 \pm 47$ minutes. The post operative hospital stay of benign and malignant tumors were $3.3 \pm 1.7$ (range 2 23) and $3.4 \pm 0.9$ (range 2 9) days, respectively (Table 4). As the post operative complications, transient hypocalcemia was in 19 cases and transient hoarseness was in 13 cases. All these patients with transient hypocalcemia and hoarseness recovered within 2 months. The permanent RLN palsy occurred in only 2 cases and there was no permanent hypocalcemia patient. Seroma occurred in 9 cases, trachea injury in 3 cases, chest wall hematoma in 4 cases, minor chyle leak 
Table 3. Type of operation

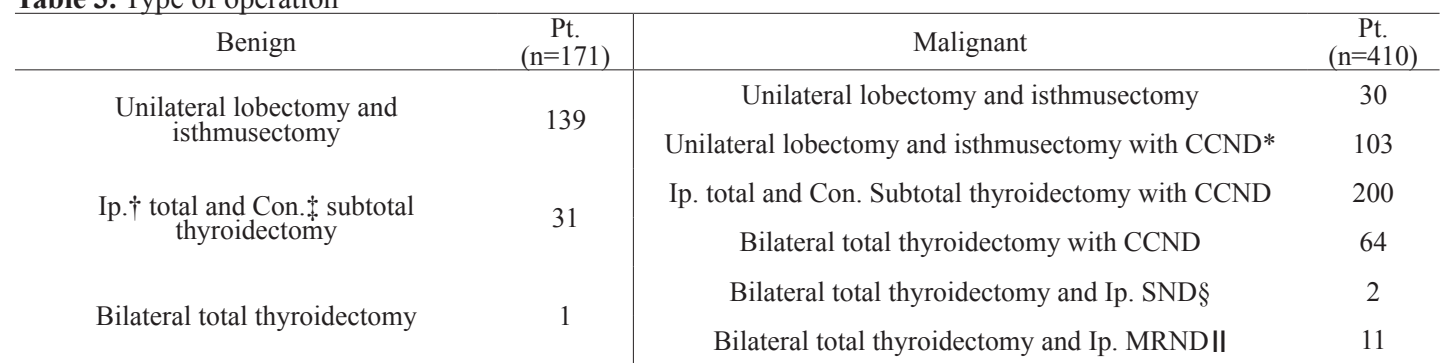

${ }^{*} \mathrm{CCND}=$ central compartment node dissection, $\dagger$ Ip. $=$ ipsilateral, $\$$ Con $=$ contralateral.

$\S \mathrm{SND}=$ selective neck dissection, $/ \mathrm{MRND}=$ modified radical neck dissection.

Table 4. Operation time and post operative hospital stay

\begin{tabular}{lcc}
\hline & Operation time (min) & Post operative hospital stay (days) \\
Benign $(\mathrm{n}=171)$ & $129.4 \pm 51.3(50 \sim 365)$ & $3.3 \pm 1.7(2 \sim 23)$ \\
Malignant $(\mathrm{n}=410)$ & & $3.3 \pm 0.8(2 \sim 9)$ \\
Unilateral lobectomy and isthmusectomy $(\mathrm{n}=133)$ & $127.7 \pm 40.0(60 \sim 249)$ & $3.4 \pm 0.9(2 \sim 9)$ \\
Ip. total and Con. subtotal thyroidectomy $(\mathrm{n}=200)$ & $133.5 \pm 47.4(55 \sim 282)$ & $3.4 \pm 0.6(2 \sim 5)$ \\
Bilateral total thyroidectomy ( $\mathrm{n}=64)$ & $160.6 \pm 49.7(90 \sim 381)$ & $5.3 \pm 1.9((3 \sim 11)$ \\
Bilateral total thyroidectomy and Ip. SND or MRND $(\mathrm{n}=13)$ & $286.9 \pm 112.7(95 \sim 485)$ & \\
\hline
\end{tabular}

Table 5. Post operative complications

\begin{tabular}{cc}
\hline Complication & Patients $(\mathrm{N})$ \\
Transient hypocalcemia & 19 \\
Transient hoarseness & 13 \\
Permanent RLN* injury & 2 \\
Seroma & 9 \\
Trachea injury & 3 \\
Esophageal injury & 1 \\
Chest wall hematoma & 4 \\
Minor chyle leak & 1 \\
\hline *RLN = recurrent laryngeal nerve &
\end{tabular}

in 1 case and esophageal injury was in 1 case (Table 5). Three cases of trachea injury and 1 case of esophageal injury were minimal point injuries caused by Harmonic Scalpel dissection and were resolved by endoscopic primary suture.

In pathologic classification of tumors, adenomatous hyperplasia was the most frequent as 107 cases in benign tumors. In malignant tumors, papillary carcinoma was 405 cases and follicular carcinoma (minimally invasive) was 5 cases. In malignancy, multiplicity and bilaterality were observed in $80(19.5 \%)$ cases, and $36(8.7 \%)$ cases, respectively. In permanent pathologic results, the mean tumor size was $2.7 \pm 1.2$ (range $0.4 \sim 6.0) \mathrm{cm}$ in benign tumor patients and $0.78 \pm 0.5$ (range 0.1 4.0) $\mathrm{cm}$ in malignant tumor patients. Among 410 malignant tumor patients, PTMC patients were $331(80.7 \%)$. All the malignant tumor patients (except 30 patients) underwent CCND, and in the cases of lateral neck node metastasis, the patients underwent MRND or SND. Among them, 112 (27.3\%) patients showed central compartment node metastasis and $13(3.1 \%)$ patients showed lateral neck node metastasis. In the cases of CCND, mean retrieved lymph node numbers are 4.6 \pm 3.2 (range 1 21), and in the case of MRND or SND, mean retrieved lymph node numbers are 18.8 6.4 (range 7 28) (Table 6).

Among 410 malignant tumor patients, T1 stage was in $259(63.1 \%)$ patients, T2 stage was in $7(1.7 \%)$ patients, and T3 stage was in $144(35.1 \%)$ patients. In the T3 stage patients, most of their tumor sizes were less than $2 \mathrm{~cm}$, but the lesions invaded the thyroid capsule. In the N stage, N0 was in $298(72.7 \%)$ patients, N1a was in $99(24.1 \%)$ patients, and N1b was in 13 (3.1\%) patients. There was no distant metastasis patient. So, the stage I was in $366(89.2 \%)$ patients, stage III was in $43(10.5 \%)$ patients, and stage IVA was in $1(0.2 \%)$ patient (Table 7$)$.

Among 77 patients who underwent bilateral total thyroidectomy, 71 patients (excluded 6 patients who were extremely low risk group) had taken RAI ablation at the $4 \sim 6^{\text {th }}$ weeks after the operation and had 
Table 6. Pathologic characteristics of patients

\begin{tabular}{|c|c|c|c|c|}
\hline \multirow[b]{2}{*}{ Pathologic classification $(\mathrm{N})$} & \multicolumn{2}{|l|}{ Benign ( $\mathrm{n}=171)$} & \multicolumn{2}{|l|}{ Malignant $(n=410)$} \\
\hline & Adenomatous hyperplasia & 107 & Papillary carcinoma & 405 \\
\hline & Follicular adenoma & 58 & ( Pure type & $398)$ \\
\hline & Hurthle cell adenoma & 2 & (Follicular variant & 7) \\
\hline & Lymphocystic thyroiditis & 4 & Follicular carcinoma $(\mathrm{MI})^{*}$ & 5 \\
\hline \multirow[t]{2}{*}{ Multiplicity (N) } & & & Single lesion & 330 \\
\hline & & & Multiple lesion & 80 \\
\hline \multirow[t]{2}{*}{ Bilaterality (N) } & & & No & 374 \\
\hline & & & Yes & 36 \\
\hline Tumor size $(\mathrm{cm})$ & \multicolumn{2}{|l|}{$2.68 \pm 1.2$ (range $0.4 \sim 6.0$ ) } & \multicolumn{2}{|l|}{$0.78 \pm 0.5$ (range $0.1 \sim 4.0$ ) } \\
\hline \multicolumn{5}{|l|}{ Retrieved lymph node $(\mathrm{N})$} \\
\hline Central compartment & \multicolumn{4}{|c|}{$4.6 \pm 3.2$ (range $1 \sim 21)$} \\
\hline Lateral neck & \multicolumn{2}{|l|}{ - } & $18.8 \pm 6.4$ (range $7 \sim 28$ ) & \\
\hline
\end{tabular}

*MI = minimally invasive.

taken ${ }^{131}$ I whole body scan in the second day after the RAI ablation. There was nobody who showed abnormal RAI uptake. 4 months after the operation, the patients' serum thyroglobulin level (TSH suppressed) was checked. Among the 77 patients, the serum thyroglobulin level of $70(90.9 \%)$ patients was $<1 \mathrm{ng} /$ $\mathrm{ml}$, and the serum thyroglobulin level of 7 (9.1\%) patients was $>1 \mathrm{ng} / \mathrm{ml}(5.6 \pm .3 .7 \mathrm{ng} / \mathrm{ml})$. These 7 patients underwent the operation in our early experience, and the elevated serum thyroglobulin level was due to the remnant thyroid tissue on the operation bed (may be the contralateral ligament of Berry area). Through the short-term regular follow-up, these patients may be performed re-treatment of RAI. All the malignant tumor patients were performed on with neck ultrasonography 10 and 18 months after the operation to check out the local recurrence. There was no recurrent tumor patient during the follow up period.

\section{Discussion}

With the development of laparoscopic operation and endoscopic instruments, various techniques of endoscopic thyroidectomy have been reported during the past decade. Shimizu [3] reported gasless videoassisted neck surgery (VANS) in 1999, Ikeda [4, 5] reported axillary endoscopic thyroidectomy in 2000, Ohgami M [6] introduced endoscopic thyroidectomy using the breast approach in 2000, and various videoassisted thyroid operation techniques have been reported $[9,10]$. For better cosmetic results, these endoscopic thyroidectomies used various approaches such as cervical, anterior chest wall, breast, axillary, axillobilateral-breast (ABBA), and bilateral axillo-breast (BABA) [3 7, 9 15]. And for the creation of working space, the continuous $\mathrm{CO}_{2}$ gas insufflation method and gasless method using an external retractor were applied. These various approaches and methods for working space have their own advantages and pitfalls, so no one can confirmatively say that which technique is better and optimal yet.

In the trans-axillary approach which Ikeda had introduced, the surgeon can approach the lateral aspect of the thyroid; from there he can easily manipulate the superior and inferior pole of the thyroid and identify the parathyroid and RLN $[4,5,11,12]$. In addition, the cosmetic result of this method is better than that of the cervical or anterior chest wall approach. This is because the operation scar remains in the axillary area which is covered when the arm is in its natural position [11]. Ikeda $[4,5,11,12]$ had used the continuous $\mathrm{CO}_{2}$ gas insufflation method for making working space. In this method, due to the relatively small working space, the operation field of view can be easily disturbed by the smoke or fume created by the uses of the electrical cautery and Harmonic Scalpel; the operation space can also be easily disrupted by the suction for bleeding or smoke clearance [13]. So to counter this, we have developed a gasless endoscopic operation method using an external retractor with the trans-axillary approach [7]. The gasless endoscopic method has no risk of complications such as hypercapnia, respiratory acidosis, tachycardia, subcutaneous emphysema, and air embolism. In the gasless endoscopic method some parts of the operation 
Table 7. TNM stage

\begin{tabular}{cc}
\hline & Patients No. (\%) \\
\hline T stage & \\
T1/ T2/ T3 & $259(63.1) / 7(1.7) / 144(35.1)$ \\
N stage & \\
N0/ N1a/ N1b & $298(72.7) / 99(24.1) / 13(3.1)$ \\
Stage & $366(89.2)$ \\
I & $43(10.5)$ \\
III & $1(0.2)$ \\
IVA & \\
\hline
\end{tabular}

procedures can be performed under the direct nakedeye view and the operation instruments used in open surgery can also be used concomitantly $[3,16]$. We have developed our own external retractor which can be connected with the continuous suction line by a canal in the midline of the retractor blade. This system can prevent operation view disturbance by the smoke or fume. In our operation method, we utilize advantages from both the gasless method and trans-axillary approach. The most remarkable advantage of this method can be focused in performance of CCND in malignant tumor patients. In this method, the surgeon makes approaching route between the SCM branches and dissects anterior surface of the carotid sheath and drops the carotid sheath just below the strap muscles. This approach enables surgeon to conduct the complete ipsilateral CCND from the carotid artery to substernal notch and prelaryngeal area including paraesophageal lymph nodes. Another benefit of this approach is that the anterior surface of SCM (sternal head) and strap muscle is not dissected. This enables the surgeon to preserve the sensory nerve around the anterior neck area and post operative hypesthesia on its region can be avoided.

Of course, there are some problems in this method. This method is more invasive and needs more operation time than open surgery due to the wide dissection from the axilla to the anterior neck area. It is also indicated that approach to the contralateral superior pole of the thyroid using this method is difficult $[4,5]$. However, we conduct subcapsular dissection of anterior thyroid surface for the making of working space until contralateral lobe is exposed. And we usually use 45-degree scope which enables us to see downward easily. There is little difficulty to identify the contralateral superior pole of thyroid.

Because the endoscopic instruments are inserted and worked through the narrow and long route from the axilla to the anterior neck area, frequent collisions of surgical instruments in the operation field (so-called "sword fighting") also can be a problem [14]. This problem can be resolved by a second skin incision $(0.5 \mathrm{~cm}$, medial side of the anterior chest wall $)$ which enables a multi-angular approach and a comfortable manipulation of endoscopic instruments [7].

Recently, these various endoscopic techniques were generally accepted as cosmetically-advantageous surgical methods for benign tumors, follicular adenoma, and some adenomatous hyperplasia [3 5, 9 12]. But as a new treatment modality in malignant tumor patients, the following problems have to be in considerations. To prevent local recurrence and distant metastasis, the safety and radicalness of the operation method should be confirmed. Many researchers doubt about the benefit of endoscopic thyroid surgery in malignant tumor patients [17]. But, the safety and radicalness of endoscopic or laparoscopic surgery in other organ malignancies were already proved [18]. They reported no differences in recurrence and survival rates between conventional open surgery and endoscopic or laparoscopic surgeries. Furthermore, endoscopic techniques have some priorities in cosmetic effect and post operative quality of life. There are various reports about endoscopic application and surgical results for PTMC in a low risk group [15, 19 23]. Miccoli et al. [20], in a prospective study, showed no difference between conventional open thyroidectomy and endoscopic thyroidectomy in surgical completeness compared to the 24-hour ${ }^{131}$ I uptake in thyroid scan and postoperative thyroglobulin. Lombardi et al. [23] reported safety and effectiveness of endoscopic thyroidectomy and selective neck dissection in papillary thyroid microcarcinoma in a low risk group. In our study, we have performed endoscopic thyroidectomy with prophylactic central compartment node dissection in 458 malignant tumor patients and experimentally tried modified radical neck or selective neck dissection according to the presence of lateral neck node metastasis in 13 patients. Afterward, we performed RAI (radio-active iodine) therapy for the indicated patients, and followed up with the RAI whole body scan, serum thyroglobulin, and neck ultrasonography. There was 
no local recurrence and distant metastasis until now. Although the follow-up period is too short to draw any conclusion of oncologic safety or prognosis, the early surgical outcomes are satisfactory.

As described above, we had some limitations in the inclusion criteria of patients. This was because we worried about the limit and safety of the endoscopic thyroidectomy. But, as more experiences accumulated, we have extended the inclusion criteria. In the size criteria, microcarcinomas were accepted according to the preoperative ultrasonography, but patients with the lesion located near the posterior capsule, especially adjacent to the tracheo-esophageal groove, were excluded. The patients who had only a few numbers of lymph node metastasis without distant metastasis and perinodal infiltration were also included. There were 7 cases of the T2 stage in tumor size, and all of them were the follicular variant of papillary carcinoma. The patients who showed multiple or bilateral lesions underwent radical resection of the thyroid. The operation time and post operative hospital stay are still a little bit longer than that of conventional open thyroidectomy, but they are decreasing as the experiences accumulate.

The Harmonic Scalpel is a vascular sealing instrument which heats up to $80^{\circ} \mathrm{C}$ through the vibration of the tip at $55,000 \mathrm{~Hz}$ and it denaturizes the tissue protein with minimal thermal trauma of the adjacent tissues. The safety and effectiveness of harmonic Scalpel were already reported in many papers [3, 24, 25]. The Harmonic Scalpel is generally used in the laparoscopic and endoscopic surgeries. However, there are some reports about permanent RLN injuries caused by the collateral energy of the Harmonic Scalpel $[26,27]$. Actually, we had 19 cases of temporary hypocalcemia and 13 cases of temporary hoarseness as postoperative complications, and all of them recovered in 2 months after the operations. We can assume that the collateral injury of the Harmonic
Scalpels can cause these complications. To avoid this injury the following factors should be acknowledged: the Harmonic Scalpel tip should be placed $2 \mathrm{~mm}$ apart from the nerve, the tip should not touch the nerve just after action, and the surgeon should not use the instrument over 20 seconds at one time [28, 29].

For the application of endoscopic thyroidectomy as a thyroid cancer treatment modality, the selection criteria of cancer patients for endoscopic thyroidectomy are most important. In other words, early cancers with low risk group should be the candidates for endoscopic thyroidectomy. Recently, many reported the experiences of endoscopic thyroid surgery for PTMC with a low risk group [15, 16, 19 23]. During the endoscopic thyroidectomy, to prevent the cancer cell disseminations and minimize the possibility of local recurrence, the safety of the operation methods and degree of surgical skill are important. In this viewpoint, we can offer gasless trans-axillary approach for endoscopic thyroidectomy as a safe and effective method. If the safety and radicalness of endoscopic thyroidectomy as PTMC treatment can be proven by performing perfect total thyroidectomies with CCND, the application objects and the development area can be gradually extended.

\section{Conclusion}

Endoscopic thyroidectomy is a valuable treatment method for benign thyroid tumor patients. It can also be selectively applied as an effective treatment modality for early thyroid cancer patients such as papillary thyroid microcarcinoma. Through technical reinforcement along with experience accumulation and development of endoscopic instruments, endoscopic thyroidectomy can overcome the limit of safety and oncologic radicalness, and can also be a new treatment modality of thyroid cancer.

\section{References}

1. Gagner M. Endoscopic subtotal parathyroidectomy in patients with primary hyperparathyroidism. Br J Surg 1996;83:875.

2. Hüscher CSG, Chiodini S, Napolitano C, Recher A. Endoscopic right thyroid lobectomy. Surg Endosc 1997;11:877.
3. Shimizu K, Akira S, Jasmi AY, Kitamura Y, Kitagawa W, Akasu H, et al. Video-assisted neck surgery: endoscopic resection of thyroid tumors with a very minimal neck wound. J Am Coll Surg 1999;188:697-703.

4. Ikeda Y, Takami H, Sasaki Y, Kan S, Niimi M. Endoscopic resection of thyroid tumors by the axillary 
approach. J Cardiovasc Surg 2000; 41: 791-792.

5. Ikeda Y, Takami H, Sasaki Y, Kan S, Niimi M. Endoscopic neck surgery by the axillary approach. $J$ Am Coll Surg 2000; 191:336-340.

6. Ohgami M, Ishii S, Arisawa Y, Ohmori T, Noga K, Furukawa T, et al. Scarless endoscopic thyroidectomy: breast approach for better cosmesis. Surg Laparosc Endosc Percutan Tech 2000; 10: 1-4.

7. Yoon JH, Park CH, Chung WY. Gasless endoscopic thyroidectomy via an axillary approach: experience of 30 cases. Surg Laparosc Endosc Percutan Tech 2006;16:226-231.

8. Greene FL, Page DL, Fleming ID, Fritz AG, Balch CM, Haller DG, Morrow M. AJCC cancer staging handbook: TNM classification of malignant tumors 6th ed. Springer-Verlag, New York. 2002:77-87.

9. Miccoli P, Berti P, Bendinelli C, Conte M, Fasolini F, Martino E. Minimally invasive video-assisted surgery of the thyroid: a preliminary report. Langenbecks Arch Surg 2000; 385: 261- 364 .

10. Gagner M, Inabnet WB, 3rd. Endoscopic thyroidectomy for solitary thyroid nodules. Thyroid 2001; 11: 161164.

11. Ikeda Y, Takami H, Sasaki Y, Takayama J, Niimi M, Kan S. Comparative study of thyroidectomies. Endoscopic surgery versus conventional open surgery. Surg Endosc 2002;16(112):1741-5.

12. Ikeda Y, Takami H, Sasaki Y, Takayama J, Kurihara H. Are there significant benefits of minimally invasive endoscopic thyroidectomy? World J Surg 2004;28:1075-8.

13. Kim JS, Kim KH, Ahn CH, Jeon HM, Kim EG, Jeon CS. A clinical analysis of gasless endoscopic thyroidectomy. Surg Laparosc Endosc Percutan Tech 2001; 11: 268-272.

14. Shimazu K, Shiba E, Tamaki Y, Takiguchi S, Taniguchi E, Ohashi S, Noguchi S. Endoscopic thyroid surgery through the axillo-bilateral-breast approach. Surg Laparosc Endosc Percutan Tech 2003;13(3):196-201.

15. Chung YS, Choe JH, Kang KH, Kim SW, Chung KW, Park KS, et al. Endoscopic thyroidectomy for thyroid malignancies: comparison with conventional open thyroidectomy. World J Surg 2007;31:2302-2306.

16. Kitagawa W, Shimizu K, Akasu H, Tanaka S. Endoscopic neck surgery with lymph node dissection for papillary carcinoma of the thyroid using a totally gasless anterior neck skin lifting method. $\mathrm{J} \mathrm{Am} \mathrm{Coll}$ Surg 2003;196:990-4.

17. Duh QY. Presidential address: minimally invasive en- docrine surgery-standard of treatment or hype? Surgery 2003;134:849-857.

18. The Clinical Outcomes of Surgical Therapy Study Group. A comparison of laparoscopically assisted and open colectomy for colon cancer. $N$ Engl J Med. 2004;350(20):2050-9.

19. Miccoli P, Berti P, Raffaelli M, Materazzi G, Baldacci $\mathrm{S}$, Rossi G, et al. Comparison between minimally invasive video-assisted thyroidectomy and conventional thyroidectomy: a prospective randomized study. Surgery 2001;130:1039-1043.

20. Miccoli P, Elisei R, Materazzi G, Capezzone M, Galleri D, Pacini F, et al. Minimally invasive video-assisted thyroidectomy for papillary carcinoma: a prospective study of its completeness. Surgery 2002;132:10701074.

21. Kitano H, Fujimura M, Kinoshita H, Kataoka H, Hirano M, Kitajima M. Endoscopic thyroid resection using cutaneous elevation in lieu of insufflation. Surg Endosc 2002;16:88-91.

22. Lombardi CP, Raffaelli M, De Crea C, Princi P, Castaldi P, Spaventa A, et al. Report on 8 years of experience with video-assisted thyroidectomy for papillary thyroid carcinoma. Surgery 2007;142:944-51.

23. Lombardi CP, Raffaelli M, Princi P, De Crea C, Bellantone R. Minimally invasive video-assisted functional lateral neck dissection for metastatic papillary thyroid carcinoma. Am J Surg 2007;193:114-118.

24. Amaral JF. The experimental development of an ultrasonically activated scalpel for laparoscope use. Surg Laparosc Endosc 1994;4:92-99.

25. Amaral JF. Laparoscopic cholecystectomy in 200 consecutive patients using an ultrasonically activated scalpel. Surg Laparosc Endosc 1995; 5: 255-262.

26. Inabnet WB 3rd, Jacob BP, Gagner M. Minimally invasive endoscopic thyroidectomy by a cervical approach. Surg Endosc 2003;17:1808-11.

27. Park YL, Han WK, Bae WG. 100 cases of endoscopic thyroidectomy: breast approach. Surg Laparosc Endosc Percutan Tech 2003;13:20-5.

28. Owaki T, Nakano S, Arimura K, Aikou T. The ultrasonic coagulating and cutting system injures nerve function. Endoscopy 2002;34:1-5.

29. Harold KL, Pollinger H, Matthews BD, Kercher KW, Sing RF, Heniford BT. Comparison of ultrasonic energy, bipolar thermal energy, and vascular clips for the hemostasis of small-, medium-, and large-sized arteries. Surg Endosc 2003; 17:1228-30. 